\title{
Cracking urban mobility
}

\author{
H. A. Carmona $\odot,{ }^{1}$ A. W. T. de Noronha $\odot,{ }^{1,2}$ A. A. Moreira, ${ }^{1}$ N. A. M. Araújo,${ }^{1,3,4}$ and J. S. Andrade, Jr. ${ }^{1, *}$ \\ ${ }^{1}$ Departamento de Física, Universidade Federal do Ceará, 60451-970 Fortaleza, Ceará, Brazil \\ ${ }^{2}$ Instituto de Ciências Exatas e da Natureza, Universidade da Integracão Internacional da Lusofonia Afro-Brasileira, \\ 62785-000 Acarape, Ceará, Brazil \\ ${ }^{3}$ Departamento de Física, Faculdade de Ciências, Universidade de Lisboa, 1749-016 Lisboa, Portugal \\ ${ }^{4}$ Centro de Física Teórica e Computacional, Universidade de Lisboa, 1749-016 Lisboa, Portugal
}

(Received 12 January 2020; accepted 1 October 2020; published 26 October 2020)

\begin{abstract}
Assessing the resilience of a road network is instrumental to improve existing infrastructures and design new ones. Here, we apply the optimal path crack model (OPC) to investigate the mobility of road networks and propose a new proxy for resilience of urban mobility. In contrast to static approaches, the OPC accounts for the dynamics of rerouting as a response to traffic jams. Precisely, one simulates a sequence of failures (cracks) at the most vulnerable segments of the optimal origin-destination paths that are capable to collapse the system. Our results with synthetic and real road networks reveal that their levels of disorder, fractions of unidirectional segments and spatial correlations can drastically affect the vulnerability to traffic congestion. By applying the OPC to downtown Boston and Manhattan, we found that Boston is significantly more vulnerable than Manhattan. This is compatible with the fact that Boston heads the list of American metropolitan areas with the highest average time waste in traffic. Moreover, our analysis discloses that the origin of this difference comes from the intrinsic spatial correlations of each road network. Finally, we argue that, due to their global influence, the most important cracks identified with OPC can be used to pinpoint potential small rerouting and structural changes in road networks that are capable to substantially improve urban mobility.
\end{abstract}

DOI: 10.1103/PhysRevResearch.2.043132

\section{INTRODUCTION}

Traffic congestion is part of the daily life in a metropolitan region. In the top 20 cities in the United States, it is estimated that the average daily commuter wastes more than $85 \mathrm{~h} / \mathrm{yr}$ in traffic congestion [1]. Boston heads this list with a $160+$ $\mathrm{h} / \mathrm{yr}$ average delay. The numbers are even worse in cities, such as Moscow, London, Bogota, or Mexico City where the average time wasted per year exceeds $200 \mathrm{~h}$. This inefficiency not only impacts on life quality and the environment, but also compromises economic growth. A recent study using data from 88 U.S. metropolitan areas suggests that a seemingly harmless average delay of $4.5 \mathrm{~min}$ for each one-way auto commute in a city is enough to slow down job growth [2].

To proper assess urban mobility, one needs to account for the impact of road congestion in global traffic [3-7]. Li et al. [8] proposed to apply percolation theory to evaluate how global connectivity is lost when vulnerable roads are congested. Their static analysis for different hours of the day gives insight into normal and rush-hour traffic and helped identifying vulnerable roads [4,9-11]. However, in reality, users are actively evaluating their routes and taking alternative paths to avoid traffic jams [12-14]. Thus, the probability that

\footnotetext{
*soares@ fisica.ufc.br
}

Published by the American Physical Society under the terms of the Creative Commons Attribution 4.0 International license. Further distribution of this work must maintain attribution to the author(s) and the published article's title, journal citation, and DOI. a road gets congested depends not only on its average level of traffic, but also on the likelihood that users take it in their route $[15,16]$.

Without central planning, travelers usually choose the route that minimizes their traveling time. However, when a road segment gets congested, a new optimal route needs to be found. The optimal path crack model (OPC) was introduced as a general framework to study the resilience of a network infrastructure to a sequence of optimal path failures $[17,18]$. The OPC is described as follows. Let us consider a square lattice of size $L$ with periodic boundary conditions in the horizontal direction and fixed boundary conditions at the top and bottom. To each link, a traveling time $t$ is randomly assigned according to a given probability distribution $P(t)$ with $t>0$. Using Dijkstra's algorithm [19], the first optimal path is identified which minimizes the total traveling time between the bottom and the top of the lattice. We then search and remove the most vulnerable link along this path, defined as the one with the highest traveling time. The next optimal path is identified, which cannot contain the removed link, and its vulnerable link is also removed. We proceed iteratively until the lattice is disrupted and no more paths can be found. The OPC is then the set of all removed links. In the limit of strong disorder, all cracks are located on a single self-similar connected line of fractal dimension equal to 1.22 [17]. As a matter of fact, this exponent value is statistically identical to the fractal dimension previously found for the optimal path line under strong disorder [20-23], "strands" in invasion percolation [20,21], paths on minimum spanning trees [24], and watersheds on uncorrelated landscapes $[18,25]$. In the case of 

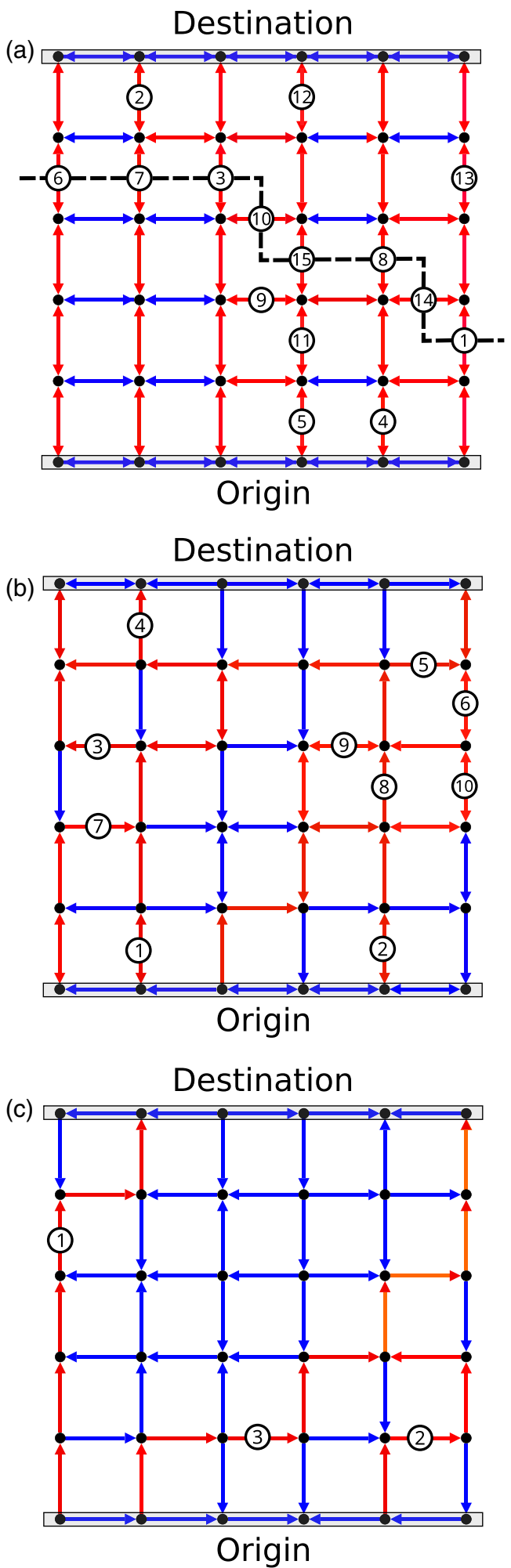

FIG. 1. Sequence of removed links during the OPC process for $6 \times 6$ square lattices under weak disorder in traveling times $(\beta=$ 0.002 ) and different values of the fraction $p$ of unidirectional links, namely, (a) $p=0$, (b) $p=0.4$, and (c) $p=1$. Before the collapse of the system, all links in red were part of an optimal path, at least, once, from the bottom (origin) to the top (destination) of the lattice. Those removed are indicated with white circles in the middle, numbered according to the OPC removal sequence as explained in the main text. The number of removed links clearly decreases with $p$. The dashed weak disorder, the cracks spread all over the entire network before global connectivity is lost so that the total number of removed links scales as $N_{r} \sim L^{d}$, where $d$ is the topological dimension of the lattice [17].

So far, all studies on OPC considered nondirected networks. However, road networks always have a non-negligible fraction of one-way roads, which is expected to substantially affect the traffic dynamics [26-28]. In addition, by contrast to previous static approaches, the fact that OPC accounts for the dynamics of rerouting as a response to traffic jams suggests that this model may be a better proxy for resilience of urban mobility. It is under this framework that here we investigate the behavior of OPC when applied to synthetic and real road networks. This paper is organized as follows. In Sec. II, we study the role of disorder and unidirectionality on the OPC model applied to synthetic road networks. In Sec. III, the OPC is applied to the real road networks of Manhattan and downtown Boston. In Sec. IV, we present the conclusions of our paper.

\section{ROLE OF DISORDER AND UNIDIRECTIONALITY ON THE OPC}

In order to better understand the combined role of disorder and unidirectionality on the OPC, we applied the model to synthetic road networks in which the links are assigned to be unidirectional with probability $p$ and bidirectional with probability $(1-p)$. For $p=0$ we recover the OPC of a fully bidirectional lattice, whereas for $p=1$ all links are unidirectional. Disorder is introduced by assigning the traveling times of unidirectional links according to a hyperbolic distribution $p\left(\tau_{i}\right) \propto 1 / \tau_{i}$, truncated between $\tau_{\max }=1$ and $\tau_{\min }=$ $\exp (-\beta)$, where $\beta \geqslant 0$ is the parameter that controls the disorder. Typical realizations of the OPC model for small networks generated with weak disorder are shown in Fig. 1. Note that each bidirectional link in the network has distinct traveling times associated with its two directions. As previously observed [17], the set of the OPC cracks generated in fully bidirectional networks $(p=0)$ always contains a contiguous subset that spans the entire system from left to right, regardless of the level of disorder. In the presence of any amount of unidirectional links $(p>0)$, however, the cracks do not necessarily form a contiguous fracture that divides the network into two pieces. Moreover, the larger the value of $p$, rarer is the occurrence of this spanning fracture. When the unidirectional links are assigned independently at random, there is always global connectivity (percolation), but sink nodes and closed loops are formed [29]. The links belonging to such a closed loop will never be part of a shortest path and, thus, the fracture does not need to be contiguous as they also contribute to separate the lattice into two pieces.

Figure 2 shows the logarithmic dependence of the number of removed links $N_{r}$ on the linear size of the lattice $L$ for lattices with sizes varying in the range $16 \leqslant L \leqslant 512$ and weak

line in (a) corresponds to the fracture backbone that is always present as a result of the OPC process applied to fully bidirectional networks $(p=0)$ [17]. 


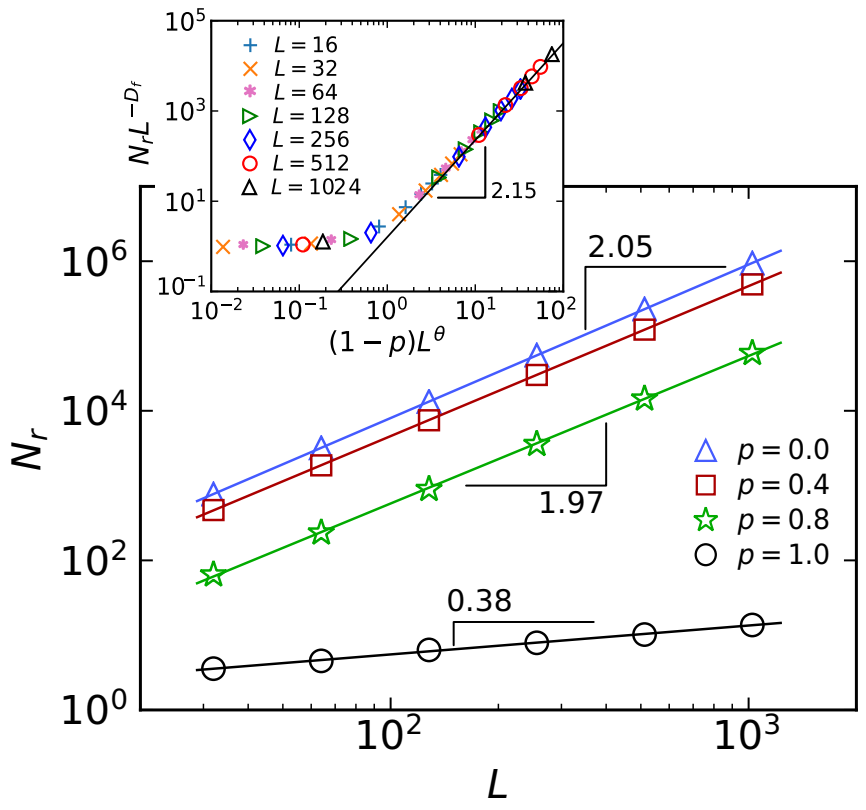

FIG. 2. Logarithmic dependence of the total number of removed links $N_{r}$ on the linear size of the lattice $L$ for different values of the fraction $p$ of unidirectional links. The symbols correspond to averages over 100 thousand network realizations and weak disorder in their traveling times $(\beta=0.002)$. The results for $p=0.4$ and 0.8 are consistent with the scaling $N_{r} \sim L^{2}$, obtained for $p=0$, namely, fully bidirectional lattices [17]. For a completely unidirectional lattice $p=1$, we find that $N_{r} \sim L^{D_{f}}$ with $D_{f}=0.382 \pm 0.002$. The error bars are smaller than the symbols. The inset shows the tricritical crossover scaling and data collapse for the OPC model on regular lattices. The scaling function is given by Eq. (1) with $D_{f}=0.38$ and $\theta=0.753$. The solid line in the inset represents a power law with exponent 2.15 .

disorder in their distribution of traveling times $(\beta=0.002)$. For $p \neq 1$, the numerical results are consistent with $N_{r} \sim L^{d}$, where $d=2$. Thus, provided that a nonzero fraction of links is bidirectional, the set of all removed links is compact as reported for networks with only bidirectional links $(p=0)$ [17]. Nevertheless, the fraction of removed links is a monotonic decreasing function of $p$, meaning that the prefactor of the power-law relation decreases too (see Fig. 2). Surprisingly, in the limit case of a completely unidirectional lattice $p=1$, we find that $N_{r} \sim L^{D_{f}}$ with $D_{f}=0.382 \pm 0.002$. Our result, therefore, indicates that the OPC set at this point belongs to a different universality class. Moreover, since $D_{f}<d$, the fraction of removed links for an infinite lattice (thermodynamic limit) is zero for $p=1$.

The statistics of the OPC set generated under weak disorder suggests that its dimension does crossover from $d$ for $p<1$ to $D_{f}$ at $p=p_{c}=1$. This crossover is analogous to what is observed at the $\theta$ point of polymer systems [30-32]. At high temperatures, the configurations of a polymer chain are well described by a self-avoiding random walk as the only relevant interactions are excluded volume. However, at the $\theta$ temperature, the attractive forces are no longer negligible, and the statistics are then different. Also, in ranked surfaces, when occupying links sequentially but suppressing global

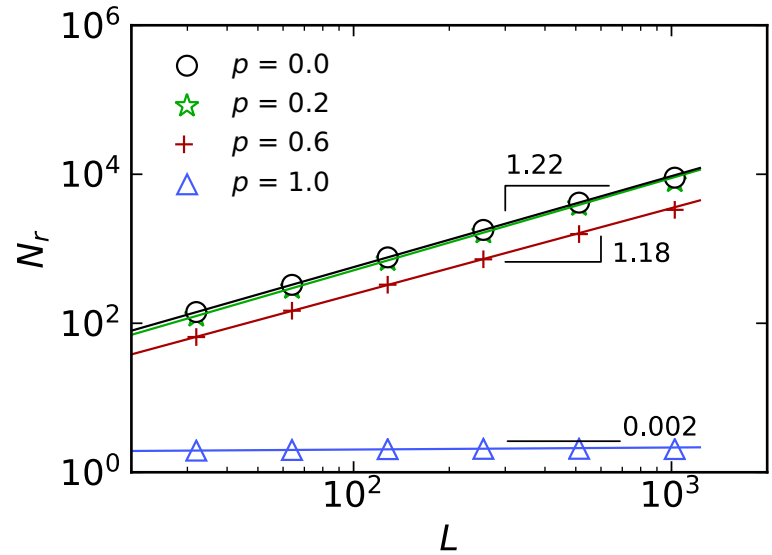

FIG. 3. Logarithmic dependence of the total number of removed links $N_{r}$ on the linear size of the lattice $L$ for different values of the fraction $p$ of unidirectional links. The symbols correspond to averages over 100000 network realizations with sizes $L=16,32$, 64,512 , and 1024 and strong disorder in their traveling times $(\beta=$ 400). The results for $p=0.4$ and 0.8 are consistent with the scaling $N_{r} \sim L_{f}^{D}$ with $D_{f}=1.22 \pm 0.01$ obtained for $p=0$, namely, fully bidirectional lattices [17]. For a completely unidirectional lattice $p=1$, we find that $N_{r} \sim L^{D_{f}}$ with $D_{f}=0.002 \pm 0.004$. The error bars are smaller than the symbols.

connectivity, the fractal dimension of the set of links that are not occupied due to this constraint changes from $3 / 4$ at the percolation threshold to 1.22 above it [25]. For the case of the $\mathrm{OPC}$, we consider the following crossover ansatz:

$$
N_{r}=L^{D_{f}} \mathcal{F}\left[\left(p_{c}-p\right) L^{\theta}\right],
$$

where $\theta$ is the crossover exponent, $\mathcal{F}[x] \sim x^{\eta}$ for $x \neq 0$ and equal to a nonzero constant at $x=0$. The inset in Fig. 2 shows the data collapse obtained with this tricritical scaling

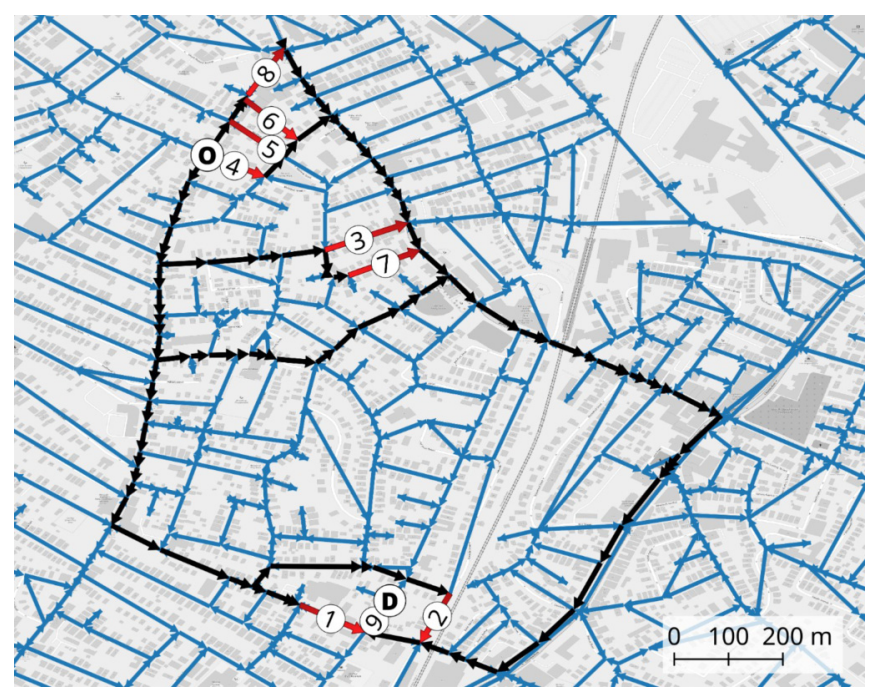

FIG. 4. Schematic representation of the OPC process for a realization of a origin $(\mathrm{O})$ destination (D) in downtown Boston. Before the collapse of the network, all links in black or red were part of an optimal path, at least, once. Those removed are in red and indicated with white circles in the middle, numbered according to the OPC removal sequence as explained in the main text. 


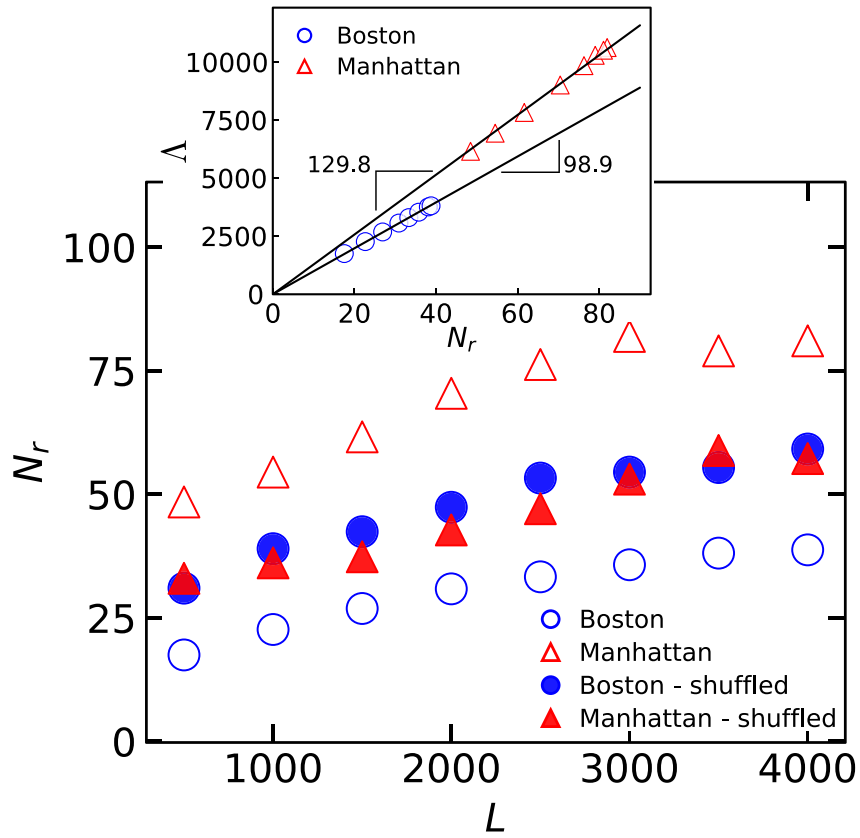

FIG. 5. Dependence of the total number of removed roads $N_{r}$ on the origin-destination distance $L$ (in meters) for downtown Boston and Manhattan (open symbols). Numerical results are averages over 2000 OD samples for each city. Also shown are the results of the OPC simulations preserving the geometry of the road networks, but shuffling the values of $t / \ell$ among randomly chosen pairs of road segments (filled symbols). The inset shows the linear dependence $\Lambda=a N_{r}$ with $a=98.9 \pm 0.03$ and $129.8 \pm 0.04$ for Boston and Manhattan, respectively. In all cases, the error bars are smaller than the symbols.

for different lattice sizes and values of $p$ with $\theta=0.753$. By fitting the power-law regime of the scaling function $\mathcal{F}$, we estimate $\eta=2.15 \pm 0.03$. From the ansatz, we expect that,

$$
D_{f}+\eta \theta=d,
$$

which is verified, within error bars. This behavior confirms that the universality class of the OPC is robust and only breaks down for a fully unidirectional lattice $(p=1)$.

For the case of local traveling times with strong disorder $\beta=400$ (see Fig. 3), the OPC results for $p \neq 1$ are also consistent with the behavior previously observed for fully bidirectional networks $(p=0)$, namely, $D_{f}=1.22 \pm$ 0.01 [17]. As in the weak disorder case, it is only for $p=1$ that the finite-size scaling of the system becomes noticeably different with $D_{f}=0.002 \pm 0.004$. A rather small number of removed links is, therefore, sufficient to block a fully directed lattice subjected to strong disorder. Summing up, our analysis with synthetic road networks shows that the higher the fraction of unidirectional segments and the level of disorder are, the lower is the resilience for urban mobility.

\section{THE OPC FOR REAL ROAD NETWORKS}

Next, we show that the OPC can be effectively used as a proxy for urban mobility. In general, the fraction of oneway roads changes from city to city. Here we apply the OPC method to two metropolitan areas in the United States,
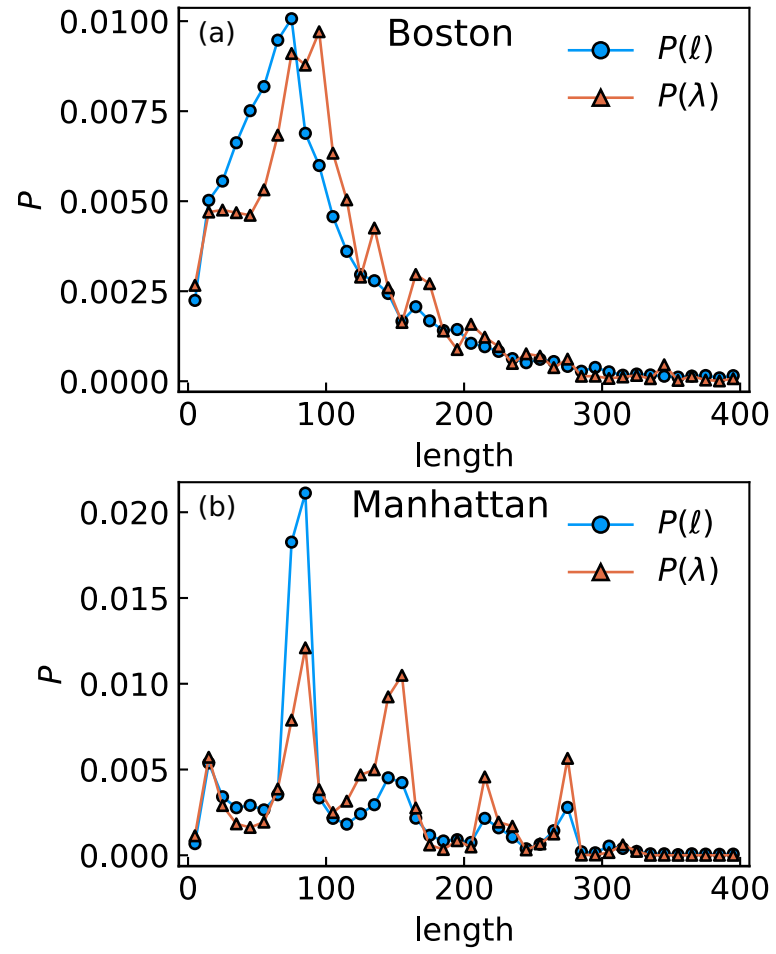

FIG. 6. Comparison between distributions of the lengths $\ell$ of all road segments, and the lengths $\lambda$ of those among all road segments that have been removed during the OPC process applied to Boston (top) and Manhattan (bottom).

namely, Manhattan and downtown Boston, the latter being the top one in the rank of North America for average time waste in traffic [1]. For this purpose, we obtained the road network structures of both urban areas from OpenStreetMap [33]. Their corresponding average traveling times per road were downloaded from Directions API Google [34], specifically for November 21th of 2018 at 4:00 a.m. This off-peak data set has been chosen because, by construction, the traveling times should somehow reflect the freeway state of the streets and avenues constituting a given urban mobility system. In order to assess the efficiency of these road networks to urban traffic, the OPC is numerically calculated for different pairs of origindestination (OD) sites. Precisely, for each OPC realization, one site of the network is selected at random to be the center of a circle of radius $L$. A point on this circle is, then, randomly chosen, and the closest road sites to the center and to this point are taken as the origin and destination, respectively, only if the distance between them is equal to $L$ within a tolerance of $5 \%$. Here, due to the fact that road segments composing the urban networks have different traveling times $t$ as well as lengths $\ell$, an optimal path is identified among all possible paths during the OPC process as the one with the minimum sum of $t / \ell$ over all its road segments.

The OPC simulations have then been performed with 2000 OD realizations for Boston and Manhattan. A typical realization of the OPC for downtown Boston is shown in Fig. 4. One should note that the bidirectional links (two-way streets and avenues) are, in fact, composed of two unidirectional ones of contrary directions that can be removed independently during the OPC process. In Fig. 5, we show how 

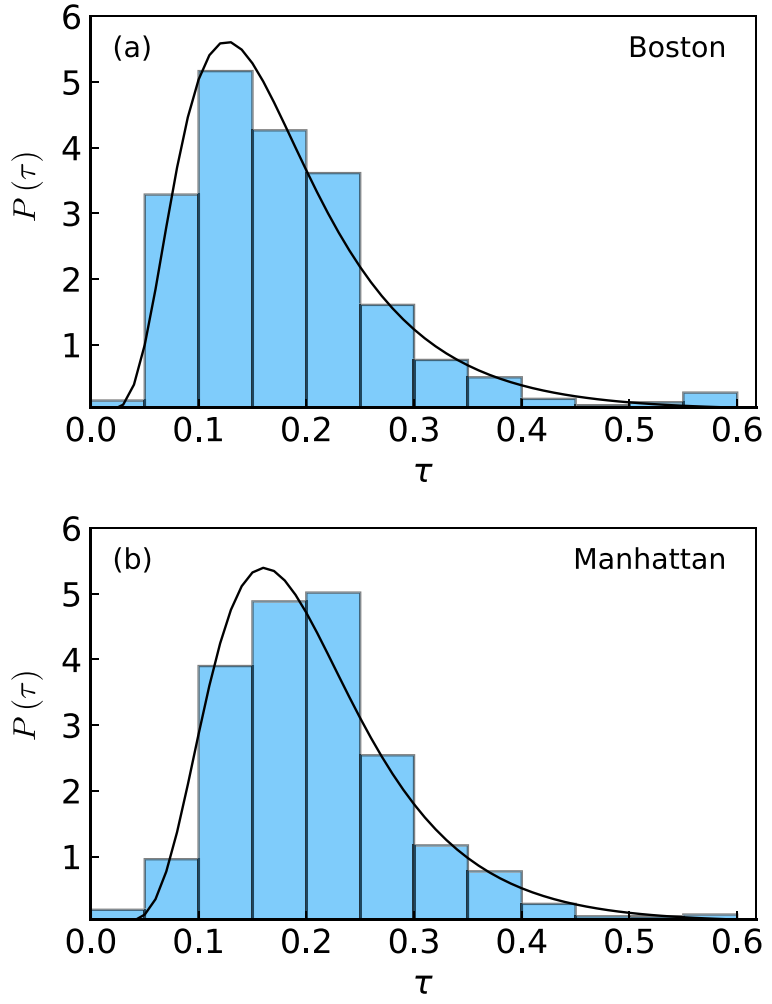

FIG. 7. Distribution $P(\tau)$ of the traveling time per length $\tau=t / \ell$ of the road segment for Boston (a) and Manhattan (b). The solid lines are best fits to a lognormal distribution with mean 0.18 and variance 0.1 for Boston and 0.21 and 0.09 for Manhattan.

the average number of removed sites $N_{r}$ varies with the distance $L$ for both urban areas. The smaller $N_{r}$ is, less resilient is the road network. These results indicate that Boston is systematically much more vulnerable than Manhattan, regardless of the origin-destination distance $L$, which is consistent with their relative positions in the national rank of urban mobility. Moreover, the same behavior can be observed if, instead of $N_{r}$, we quantify the result of each OPC process in terms of the $\operatorname{sum} \Lambda=\sum_{i=1}^{N_{r}} \lambda_{i}$, where $\lambda_{i}$ is equal to the length $\ell$ of the removed road segment at iteration $i$. Interestingly, as shown in the inset of Fig. 5, these two measures are, in fact, linearly related $\langle\Lambda\rangle=a N_{r}$ with $a=98.9$ and $129.8 \mathrm{~m}$ for Boston and Manhattan, respectively. For all practical purposes, this indicates the absence of spatial statistical correlations in the OPC process of selecting $\lambda$ from the distribution of segment lengths $P(\ell)$ of both road networks. As shown in Fig. 6, the distributions $P(\ell)$ and $P(\lambda)$ for a given road network display similar qualitative features in shape. More important, these results for Boston and Manhattan indicate that the variable $\lambda$ is selected by the OPC process over the entire range of $\ell$ values. One should note that, although the OPC process tends to pick larger values of $\lambda$ from $P(\ell)$ more frequently, this does not imply the presence of statistical correlations among links in the road networks. Such a behavior clearly reflects a local feature of the network that is captured by the OPC but cannot explain the substantial differences in the resilience of Boston and Manhattan since the OPC results for both road networks follow the same tendency.

We now investigate the origin of the substantial difference between the resilience of Boston and Manhattan. Figure 7 shows the distributions of traveling time per segment length $P(t / \ell)$ obtained for their corresponding road networks. Clearly, the disorder is weak in both cases, and the modest difference between them is not compatible with the large discrepancy observed in their resilience. Furthermore, the

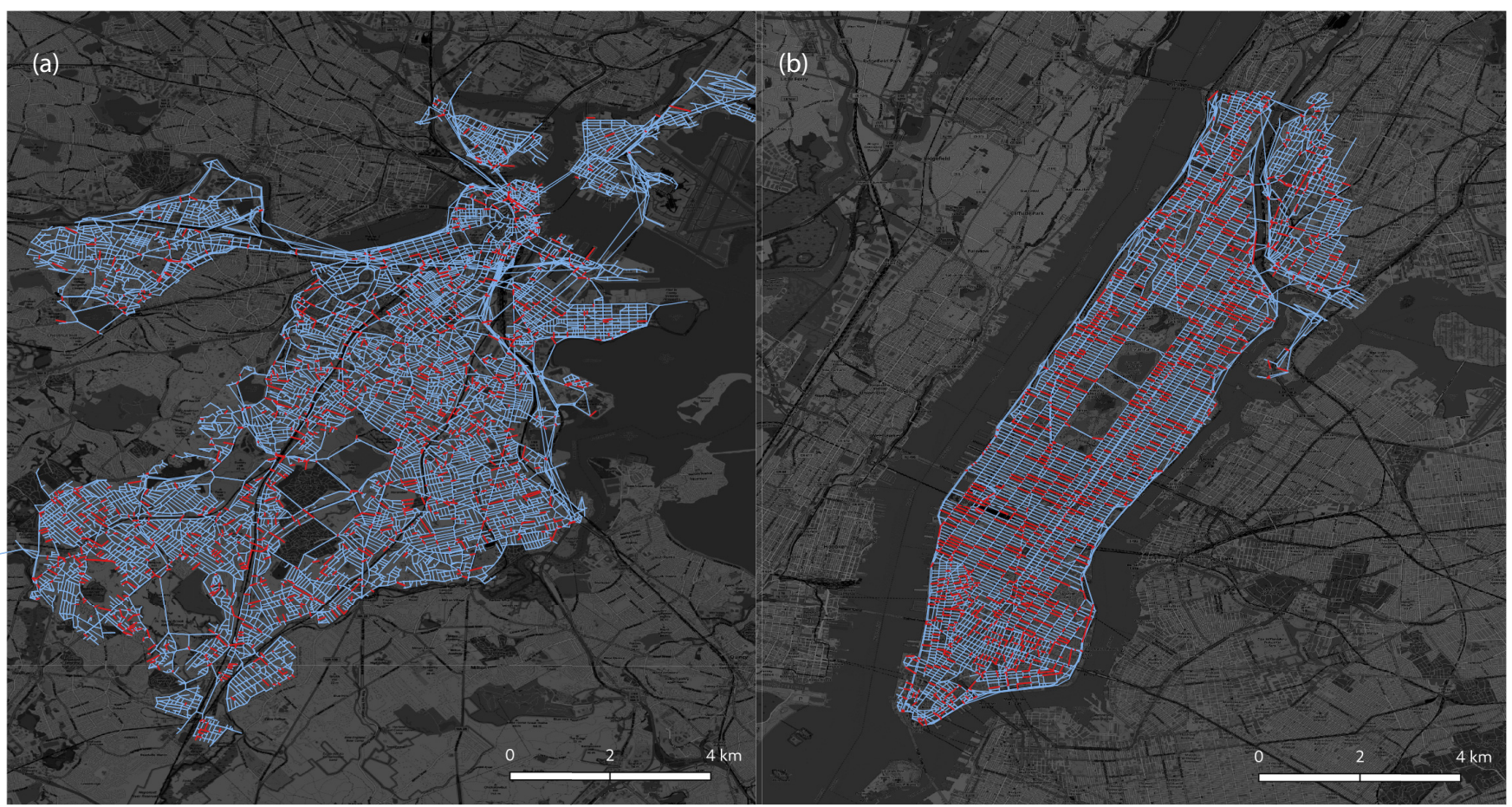

FIG. 8. Road maps for downtown Boston (left) and Manhattan (right). The thick red lines are the road segments that have been removed first. All other road segments are shown in blue. Only the OPC samples performed with OD distances $L=2000 \mathrm{~m}$ have been considered. 


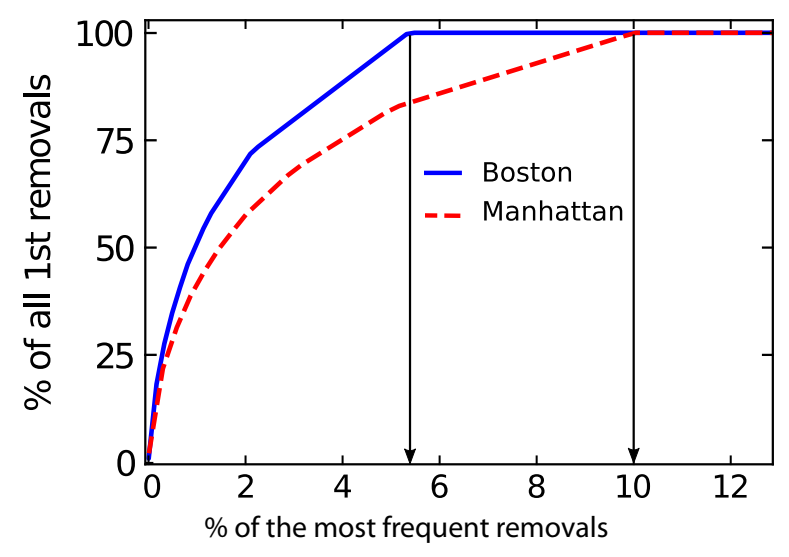

FIG. 9. Cumulative dependence of the percentage of all first removals during the OPC process on the fraction of the most frequent ones. The vertical solid lines indicate that the first removals correspond to $5.4 \%$ of all road segments in the case of Boston, whereas $10.0 \%$ is the percentage required for Manhattan.

fraction of unidirectional links $p$ found for downtown Boston is 0.65 , whereas the value for Manhattan is 0.78 . However, our results from synthetic road networks indicate that, for similar levels of disorder, the resilience of the system should decrease with the value of $p$. As a consequence, the observed difference in the resilience of Boston and Manhattan also cannot stem from their distinct $p$ values. One, therefore, can only rely on the particular features of the intrinsic spatial correlations that should be present in these urban systems to justify their very distinct responses. In order to test for this hypothesis, we performed additional OPC simulations preserving the geometry of both networks, but shuffling the values of $t / \ell$ among randomly chosen pairs of road segments. The results presented in Fig. 5 are rather surprising and twofold. First, they show that the effect of suppressing the spatial correlations is to practically collapse the curves $N_{r}$ against $L$ of both cities to a single curve, therefore, demonstrating the generality and comparative power of the OPC method for urban mobility. Second, the fact that the values of $N_{r}$ systematically increase for Boston and decrease for Manhattan as compared to the results using the real (nonshuffled) data sets corroborate the ability of our approach to properly capture negative and positive effects of spatial correlations on urban mobility.
One question that naturally arises is how the OPC method can be used to enhance urban mobility. A possible answer is to prioritize rerouting and structural improvements based on the identification of those road segments that are more frequently appearing among the first removals in all OPC sequences. In Fig. 8, are the road maps for the two urban areas where the highlighted road segments (thick and red) correspond to all those removed first, considering all OPC samples performed with OD distances $L=2000 \mathrm{~m}$. For downtown Boston [see Fig. 8(a)], we find that only a percentage of $5.4 \%$ of all road segments is removed, whereas $10.0 \%$ is the value for Manhattan [see Fig. 8(b)]. The cumulative dependence of the percentage of all first removals on the percentage of the most frequent ones is shown in Fig. 9. These results suggest that, as compared to Manhattan, a relatively small number of potential local changes in Boston might be very efficient in improving urban mobility. The fact that Manhattan is much more resilient should, therefore, increase the costs of improvements.

\section{CONCLUSIONS}

To summarize, we proposed a proxy for resilience of urban mobility based on OPC. Our results with synthetic and real road networks suggest that their vulnerability to traffic congestion are strongly dependent on the level of disorder, fraction of unidirectional segments, and intrinsic spatial correlations. These observations have practical implications in the design and restructuring for improved urban mobility. With the OPC, we obtain the list of most vulnerable links, defined as the ones with the highest traveling time along the sequence of shortest origin-destination paths. The vulnerability of such a link might stem from many design factors, such as the capacity of the road, speed limit, number of crossroads, or traffic-light dynamics. We conclude that the OPC is a general and powerful method to access urban mobility and gives practical insight that can effectively help identifying and mitigating vulnerabilities of real road networks.

\section{ACKNOWLEDGMENTS}

We gratefully acknowledge financial support from the Brazilian agencies $\mathrm{CNPq}, \mathrm{CAPES}$, and FUNCAP, the National Institute of Science and Technology for Complex Systems in Brazil, and from the Portuguese Foundation for Science and Technology (FCT) under Contracts No. UIDB/00618/2020 and No. UIDP/00618/2020.
[1] T. Reed and J. Kidd, Techical Report, IRIX Research, United States, 2018 (unpublished).

[2] M. Sweet, Urban Studies 51, 2088 (2014).

[3] R. Louf and M. Barthelemy, Sci. Rep. 4, 5561 (2014).

[4] S. Colak, A. Lima, and M. C. González, Nat. Commun. 7, 10793 (2016).

[5] A. Solé-Ribalta, S. Gómez, and A. Arenas, Networks Spat. Econ. 18, 33 (2018).

[6] H. Barbosa, M. Barthelemy, G. Ghoshal, C. R. James, M. Lenormand, T. Louail, R. Menezes, J. J. Ramasco, F. Simini, and M. Tomasini, Phys. Rep. 734, 1 (2018).
[7] M. Barthelemy, Nat. Rev. Phys. 1, 406 (2019).

[8] D. Li, B. Fu, Y. Wang, G. Lu, Y. Berezin, H. E. Stanley, and S. Havlin, Proc. Natl. Acad. Sci. USA 112, 669 (2015).

[9] M. C. González, C. A. Hidalgo, and A.-L. Barabási, Nature (London) 453, 779 (2008).

[10] L. E. Olmos, S. Çolak, S. Shafiei, M. Saberi, and M. C. González, Proc. Natl. Acad. Sci. USA 115, 12654 (2018).

[11] G. Zeng, D. Li, S. Guo, L. Gao, Z. Gao, H. E. Stanley, and S. Havlin, Proc. Natl. Acad. Sci. USA 116, 23 (2019).

[12] S. Zhu and D. Levinson, PLoS One 10, e0134322 (2015). 
[13] A. Lima, R. Stanojevic, D. Papagiannaki, P. Rodriguez, and M. C. Gonzalez, J. R. Soc., Interface 13, 20160021 (2016).

[14] L. Zhang, G. Zeng, D. Li, H. J. Huang, H. E. Stanley, and S. Havlin, Proc. Natl. Acad. Sci. USA 116, 8673 (2019).

[15] P. Wang, T. Hunter, A. M. Bayen, K. Schechtner, and M. C. González, Sci. Rep. 2, 1001 (2012).

[16] S. Guo, D. Zhou, J. Fan, Q. Tong, T. Zhu, W. Lv, D. Li, and S. Havlin, EPJ Data Sci. 8, 28 (2019).

[17] J. S. Andrade, E. A. Oliveira, A. A. Moreira, and H. J. Herrmann, Phys. Rev. Lett. 103, 225503 (2009).

[18] E. A. Oliveira, K. J. Schrenk, N. A. M. Araújo, H. J. Herrmann, and J. S. Andrade, Phys. Rev. E 83, 046113 (2011).

[19] E. W. Dijkstra, Numer. Math. 1, 269 (1959).

[20] M. Cieplak, A. Maritan, and J. R. Banavar, Phys. Rev. Lett. 72, 2320 (1994).

[21] M. Cieplak, A. Maritan, and J. R. Banavar, Phys. Rev. Lett. 76, 3754 (1996).

[22] M. Porto, S. Havlin, S. Schwarzer, and A. Bunde, Phys. Rev. Lett. 79, 4060 (1997).

[23] M. Porto, N. Schwartz, S. Havlin, and A. Bunde, Phys. Rev. E 60, 2448(R) (1999).
[24] R. Dobrin and P. M. Duxbury, Phys. Rev. Lett. 86, 5076 (2001).

[25] K. J. Schrenk, N. A. M. Araújo, J. S. Andrade, and H. J. Herrmann, Sci. Rep. 2, 348 (2012).

[26] N. Schwartz, R. Cohen, D. ben-Avraham, A.-L. Barabási, and S. Havlin, Phys. Rev. E 66, 015104(R) (2002).

[27] L. Buzna, K. Peters, and D. Helbing, Physica A 363, 132 (2006).

[28] A. D. Sánchez, J. M. López, and M. A. Rodríguez, Phys. Rev. Lett. 88, 048701 (2002).

[29] A. W. T. de Noronha, A. A. Moreira, A. P. Vieira, H. J. Herrmann, J. S. Andrade, and H. A. Carmona, Phys. Rev. E 98, 062116 (2018).

[30] P.-G. de Gennes, Scaling Concepts in Polymer Physics (Cornell University Press, Ithaca, New York, 1979).

[31] I. Chang and A. Aharony, J. Phys. I 1, 313 (1991).

[32] P. H. Poole, A. Coniglio, N. Jan, and H. E. Stanley, Phys. Rev. B 39, 495 (1989).

[33] OpenStreetMap contributors, Planet dump retrieved from https: //planet.osm.org, https://www.openstreetmap.org (2019)

[34] Google, Google Directions API, https://developers.google.com/ maps/documentation/directions/start (2019) 